\title{
A rare presentation of a large goiter with papillary thyroid microcarcinoma as a chest wall mass: A case report
}

\author{
ZHEN-NING TANG, QI-LUN LIU, ZHI-QIANG HU and LI-GANG WU \\ Department of Oncological Surgery, The General Hospital of Ningxia Medical University, \\ Yinchuan, Ningxia 750004, P.R. China
}

Received August 4, 2015; Accepted December 11, 2015

DOI: $10.3892 / \mathrm{mco} .2016 .730$

\begin{abstract}
Nodular goiter affects numerous individuals worldwide. As the thyroid enlarges, it normally extends into the mediastinum as a result of its anatomical location under the investing layer of the deep cervical fascia. The present study reports the rare case of a 76-year-old man who suffered from a goiter with papillary thyroid microcarcinoma, who presented as a subcutaneous partially cystic with solid areas lesion over the chest. The patient underwent surgery and on histopathological examination, the predominant mass was characterized as a multinodular goiter with hemorrhage, necrosis and cystic change. A papillary thyroid microcarcinoma with a diameter of $0.2 \mathrm{~cm}$ was identified. The present study demonstrated a rare, to the best of our knowledge, presentation of a benign multinodular goiter in this way.
\end{abstract}

\section{Introduction}

Nodular goiter is the most common pathology of the thyroid gland. The prevalence of goitre nodules may depend on several factors, such as genes and low iodine intake (1). The majority of focal thyroid lesions are of a benign nature and the incidence of occult malignancy within a multinodular goiter ranged between 10 and $35 \%$ in the previous surgical series. The majority of nodular goiter present as an asymptomatic enlargement of the thyroid gland, or increasing symptoms of compression (2). As the thyroid enlarges, it normally extends into the mediastinum as a result of its anatomical location under the investing layer of the deep cervical fascia. The present study reported a case of a goiter with papillary thyroid microcarcinoma, which presented as a subcutaneous partially cystic with solid areas lesion in the neck extending over the chest.

Correspondence to: Professor Qi-Lun Liu, Department of Oncological Surgery, The General Hospital of Ningxia Medical University, 804 Shengli Street, Yinchuan, Ningxia 750004, P.R. China

E-mail: liuq16311@126.com

Key words: nodular goiter, thyroid cancer

\section{Case report}

A 76-year-old man of Hui ethnicity presented with swelling in the front of the neck, which had been present since the age of 42. The swelling began in the right lobe of the thyroid region and was initially small in size, however, gradually increased to the present size. Upon examination, partially cystic with solid areas swellings extended from the right side of the neck to the chest wall. The swellings were immobile and failed to move with deglutition. The cervical lymph nodes were not enlarged (Fig. 1).

Neck and chest X-rays revealed a markedly increased soft tissue density in the neck region extending to the upper sternal region and indenting the trachea (data not shown). An ultrasound of the neck revealed a disappearance of the normal right lobe of the thyroid and identified multiple hypoechoic masses with anechoic areas extending from the level of the carotid to the mid-sternum (data not shown). Inhomogeneous interior echoes were observed in the mass (data not shown). The mass on the chest wall appeared as a low echo mass with a clear margin. The lesion of the chest wall appeared to arise from thyroid tissue. Computed tomography revealed multiple coalescing cystic lesions with fluid attenuation in the subcutaneous plane of the neck and chest wall, and below the thoracic inlet. The cystic lesions appeared to be interlinked with the right thyroid lobe and only part of the left thyroid lobe was visible (Fig. 2).

The patient underwent surgery and under general anesthesia, an extended Kocher's horizontal fusiform incision was made over the neck swelling, and the middle part of the incision was extended vertically downward over the chest wall swelling. The fluid in the cyst was brown in color. The mass in the right neck was $15 \times 12 \mathrm{~cm}$ in size, ranging up to the jaw, outside to the front edge of the trapezius, down over the clavicle, and connecting with the sternum and chest wall mass. On the right side, the mass was adhered to the internal jugular vein, requiring ligation of the internal jugular vein. The mass in the chest wall was $17 \times 13 \mathrm{~cm}$ and the substernal goiter descended into the anterior mediastinum. The maximum length of the part of the mass, which had descended into the chest was $10 \mathrm{~cm}$. Upon histopathological examination, a papillary thyroid microcarcinoma with a diameter of $0.2 \mathrm{~cm}$ was identified. The predominant mass was characterized as a multinodular goiter with hemorrhage, necrosis and cystic change. The patient recovered completely within 2 weeks following surgery. 

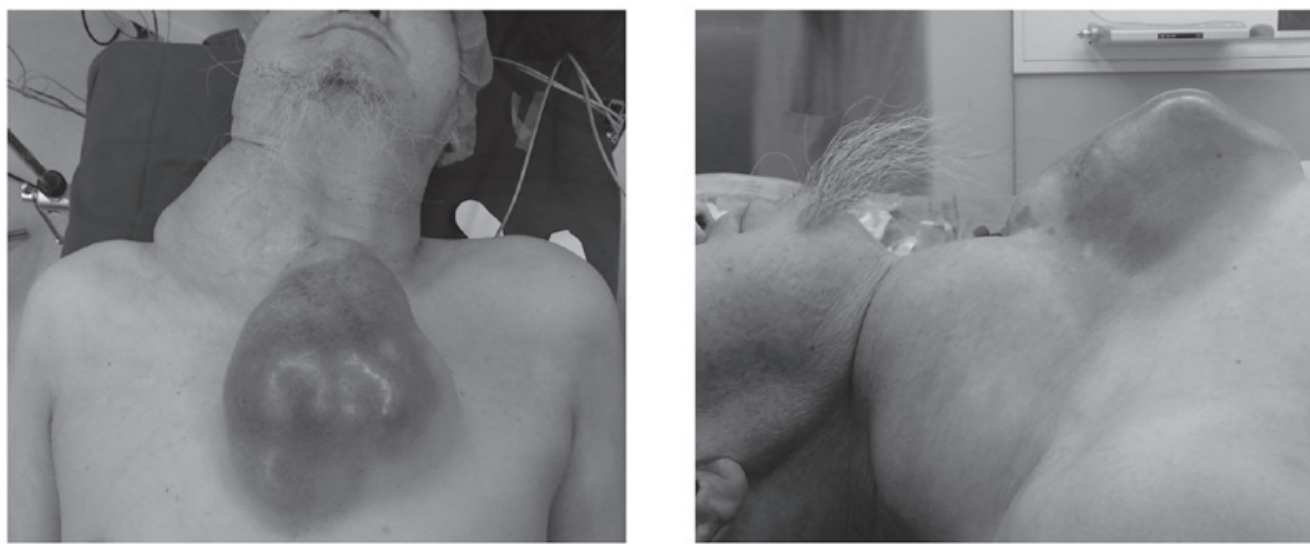

Figure 1. Preoperative photo of the patient with partially cystic with solid area swellings in the neck and chest.

A

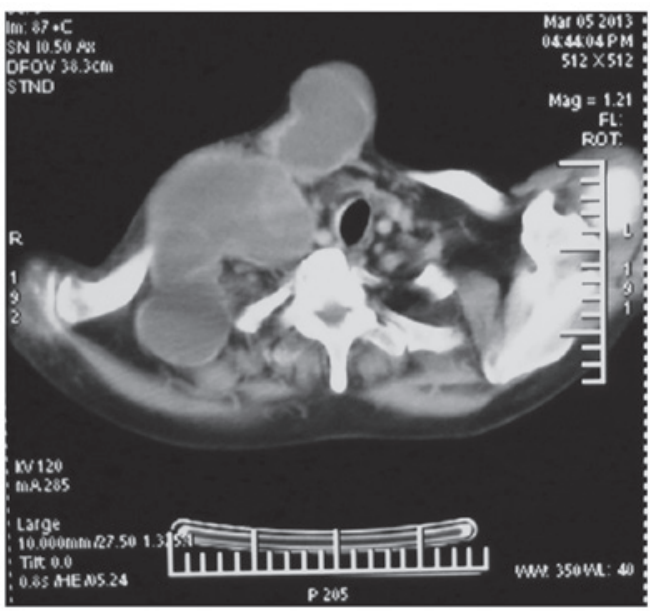

C

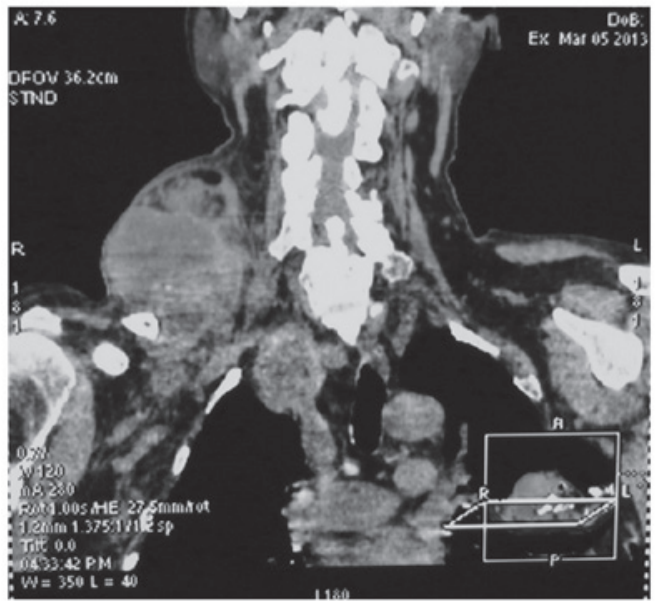

B

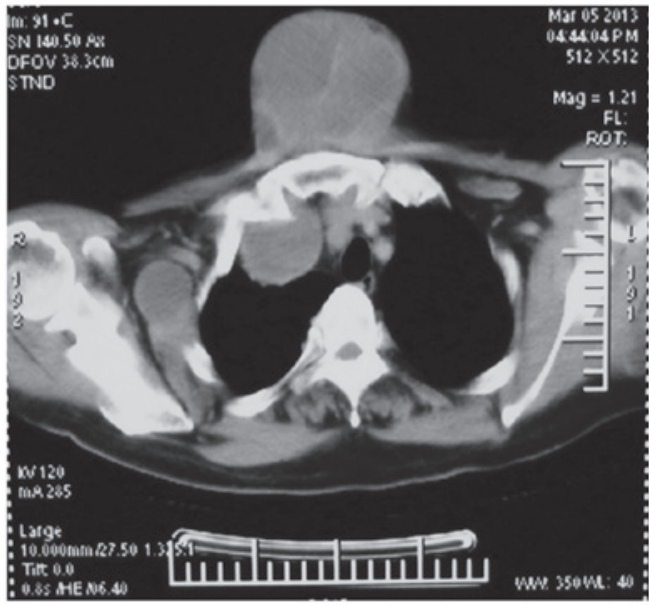

D

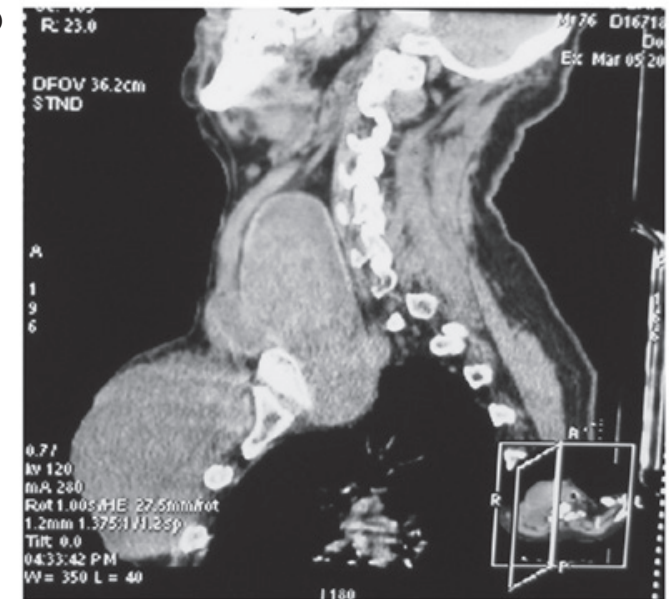

Figure 2. Computer tomography image showing multinodular goiter of the neck and chest. (A and B) Axial view; (C) coronal view; (D) sagittal view.

\section{Discussion}

The most common benign cervical and chest wall cysts are lymphangiomas, dermoid cysts, teratomas and inflammatory cysts. Multinodular goiter progresses from a diffuse enlargement of the thyroid to a multinodular structure with advancing age. It is very commonly observed that the long-standing goiter descends into the upper mediastinum space causing compression of the trachea and other cervical structures (3). A paucity of literature addressing thyroid carcinomas, which present as a chest wall mass, exist $(4,5)$. In addition, it is extremely rare for a benign multinodular goiter to present in this way. The present case report suggested that multinodular goiters may invade adjacent structures and manifest as malignancies.

\section{Acknowledgements}

The present study was supported by a grant from the Health and family planning commission of Ningxia Hui Autonomous Region (no. 2013104). 


\section{References}

1. Carle A, Krejbjerg A and Laurberg P: Epidemiology of nodular goitre. Influence of iodine intake. Best Pract Res Clin Endocrinol Metab 28: 465-479. 2014.

2. Nixon IJ and Simo R: The neoplastic goitre. Curr Opin Otolaryngol Head Neck Surg 21: 143-149, 2013.

3. Silva MN, Rubio IG, Romão R, Gebrin EM, Buchpiguel C, Tomimori E, Camargo R, Cardia MS and Medeiros-Neto G: Administration of a single dose of recombinant human thyrotrophin enhances the efficacy of radioiodine treatment of large compressive multinodular goitres. Clin Endocrinol (Oxf) 60 300-308, 2004
4. Gertz R, Sarda R and Lloyd R: Follicular thyroid carcinoma presenting as a massive chest wall tumor. Endocr Pathol 24: 20-24, 2013.

5. Patil VS, Vijayakumar A and Natikar N: Unusual presentation of cystic papillary thyroid carcinoma. Case Rep Endocrinol 2012: 732715, 2012. 